\title{
Does bright light have an anxiolytic effect? - an open trial Shawn D Youngstedt* ${ }^{*}, 2$ and Daniel F Kripke 3
}

Address: ${ }^{1}$ Department of Exercise Science, Norman J. Arnold School of Public Health University of South Carolina, Columbia, SC 29208 USA, ${ }^{2}$ Department of Psychiatry, Dorn VA Medical Center, Columbia, South Carolina, 29209, USA and ${ }^{3}$ Department of Psychiatry and Sam and Rose Stein Institute for Research on Aging, University of California, San Diego, La Jolla, CA 92093-0667, USA

Email: Shawn D Youngstedt* - syoungstedt@sc.edu; Daniel F Kripke-dkripke@ucsd.edu

* Corresponding author

Published: 30 October 2007

BMC Psychiatry 2007, 7:62 doi:10.1186/147I-244X-7-62
Received: 16 May 2007

Accepted: 30 October 2007

This article is available from: http://www.biomedcentral.com/l47/-244X/7/62

(c) 2007 Youngstedt and Kripke; licensee BioMed Central Ltd.

This is an Open Access article distributed under the terms of the Creative Commons Attribution License (http://creativecommons.org/licenses/by/2.0), which permits unrestricted use, distribution, and reproduction in any medium, provided the original work is properly cited.

\begin{abstract}
Background: The aim of this open trial was to examine the influence of acute bright light exposure on anxiety in older and young adults.

Methods: This study was ancillary to a complex 5-day laboratory experiment testing phaseresponses to light at all times of the day. On 3 consecutive days, participants were exposed to bright light (3,000 lux) for 3 hours. The Spielberger State-Trait Anxiety Inventory (Form YI) was administered 5 minutes before and 20 minutes after each treatment. Mean state anxiety before and after treatment were analyzed by age, sex, and time ANOVA. To avoid floor effects, only participants with baseline STAI levels of $\geq 25$ were included.
\end{abstract}

Results: A significant anxiolytic effect of bright light was found for the mean data, as well as for each of the three days. No significant main effect of age, sex, or interaction of these factors with STAl change were found.

Conclusion: The results show consistent and significant (albeit modest) anxiolytic effects following acute bright light exposure in low anxiety adults. Further randomized, controlled trials in clinically anxious individuals are needed.

\section{Background}

Anxiety is the most common mental illness in the US $[1,2]$. Moreover, complaints of anxiety are common among healthy individuals and have been associated with numerous negative health consequences [3-5], absenteeism [6], and decreased work productivity $[6,7]$.

Anxiolytic drugs have limited long-term efficacy. Their adverse side effects include dependency (for benzodiazepine agonists), drowsiness [8], impaired cognition and memory [8-10], and sexual dysfunction $[10,11]$. Alternative or adjuvant treatments for anxiety might be valuable.
Bright light exposure would be a potentially attractive anxiolytic treatment. The antidepressant effects of bright light are well-established for winter depression $[12,13]$, as well as many other types of nonseasonal depression [1417]. The common co-morbidity $[5,18]$ and neurochemical similarity between anxiety and depression (e.g., responsiveness to the same drugs) [19] provide rationales for expecting that bright light might also have substantial anxiolytic effects.

Although there has been little systematic investigation of the anxiolytic effect of bright light, there is some experimental support for this effect. For example, in research of 
light treatment for winter depression, anxiety-related symptoms have been reduced to approximately the same degree as depressive symptoms [20]. Similar reduction in anxiety and depression symptoms following light exposure have also been noted in healthy individuals and individuals with subsynromal SAD [21]. Some evidence suggests that both anxiolytic and antidepressant effects of light might be mediated by serotonergic mechanisms [22]. The primary aim of the present study was to explore the anxiolytic effect of bright light in a large sample. Secondary aims were to contrast effects in older (ages 60-75 yrs) vs. young (ages 18-30 yrs) adults and in women vs. men. The study was ancillary to a complex 5-6 day laboratory protocol, which allowed us to examine this effect on 3 consecutive days. The sample generally had low levels of depression, which allowed exploration of anxiolytic effects independent of antidepressant effects of bright light.

\section{Methods}

Older adults ages $60-75 \mathrm{yr}(66.6 \pm 4.5 \mathrm{yr})$ and young adults ages $18-30 \mathrm{yrs}(23.4 \pm 3.8 \mathrm{yr})$ were recruited by word of mouth, referrals, announcements in local media, and advertising in newspapers, adult fitness centers, and senior pages. Initial inclusion criteria, based on several screening questionnaires, required good health and regular participation in vigorous aerobic exercise. Exclusions included: (1) having $>1$ of the major risk factors for coronary artery disease [23] including family history of early myocardial infarction, current smoking, hypertension, history of hypercholesterolemia, and diabetes mellitus; (2) having any major symptoms or signs of cardiopulmonary disease [23], including chest pain, dizziness or syncope, orthopnea or nocturnal dyspnea, ankle edema, heart papitations, claudication, serious heart murmur, and excessive shortness of breath; (3) recent shift-work (previous 2 months) or travel across multiple time zones (previous 4 weeks); (4) abnormal sleep-wake schedule; (5) depression; (6) use of alpha or beta blockers, antihypertensives, or antidepressants; (7) or any health or mental condition that would contraindicate participating in the rigors of the experiment.

Prior to participation in the study, written informed consent was obtained from each participant, as approved by the UCSD Institutional Review Board. Included in the consent was a description that the study would include assessment of the influence of bright light on mood, which is the focus of the present report. The research was conducted in compliance with the Helsinki Declaration.

Once recruited, participants were required to pass a medical interview and further medical screening. Exclusions included elevations in fasting plasma lipids and glucose, hypertension, abnormal resting 12-lead EKG, and signs of heart disease during a physician-supervised treadmill test to volitional exhaustion. Participants were not given a psychiatric interview.

\section{Laboratory protocol}

The 5-6 day laboratory protocol was designed to examine the influence of bright light or exercise on the circadian system. Participants were randomized to bright light or exercise treatment, but the present report is limited to data associated with the bright light treatment. Each participant stayed in a studio-apartment room and followed a 90 minute "ultra-short" sleep wake cycle [24], involving 60 min wake intervals in dim light $(<50$ lux $)$ and $30 \mathrm{~min}$ sleep intervals in darkness. This cycle was repeated around-the-clock throughout the 5-day period. Food and drink were available ad libitum (excluding caffeine and alcohol). The protocol included around-the-clock collection of urinary and saliva samples (at 90 min intervals) and completion of several visual analogue scales at 4-hr intervals. Tests of retinal circadian rhythms (e.g., electrooculography, visual threshold) were performed around the clock on a subset of participants $(\mathrm{n}=12)$. During the wake periods, participants were free to engage in sedentary activities, including watching television, reading, receiving visitors, etc. Strenuous exercise was prohibited. Participants completed the Center for Epidemiological Studies Depression Scale (CESD) [25] on the first and last days of the laboratory study.

\section{Light treatments}

Participants were exposed to $3 \mathrm{hr}$ of bright light $(3,000$ lux) on 3 consecutive days. The treatment was administered via overhead cool-white fluorescent lights which distributed the light levels evenly throughout the room. The light was administered at one of 8 randomly assigned times-of-day or night, but was provided at the same timeof-day or night across the 3 days for each individual. The treatments were administered in a phase-response curve experiment establishing the direction and magnitude of phase-shift depending upon the time of treatment.

\section{Anxiety assessement}

Anxiety was assessed with Spielberger's State-Trait Anxiety Inventory (STAI; Form Y1, i.e., "how you feel at the moment") [26] at $5 \mathrm{~min}$ before and $20 \mathrm{~min}$ following all three light treatments. The reliability and validity of the STAI are well established [26]. Participants were given standardized instructions prior to completing the STAI questionnaire.

\section{Data analysis}

To avoid floor effects, an a priori decision was made to exclude participants for whom baseline STAI were $<25$, which would be approximately one standard deviation below population norms [26]. Data were averaged across 
the 3 days of assessment, and analyzed by repeated measures ANOVA, comparing responses by age group and sex. In addition, ANOVAs were calculated for each of the 3 individual days of assessment. Normal distribution of the data was established. Effect size was calculated by subtracting the mean post-light STAI from the mean pre-light STAI and dividing this difference by the pooled standard deviation. Since analysis indicated that there was no significant influence of time-of-day on baseline STAI levels or on anxiolytic responses to bright light, data were collapsed across all times of testing in the present report. Comparison of anxiolytic response between individuals whose baseline STAI levels were within the range found for clinical populations $(=48)[26]$ vs. individuals with lower STAI levels was made via an independent t-test. Normal distribution of the data was verified.

\section{Results}

The number of participants excluded from analysis due to low baseline STAI levels was 25. The present analyses included 79 participants out of a total of 104 . Four participants had clinical levels of baseline STAI $(50.8 \pm 0.87)$ (though none had formal DSM IV diagnoses of anxiety disorders), whereas 75 participants had lower levels (31.0 $\pm 0.57)$.

The older participants $(\mathrm{n}=33)$ were ages $59-75$ years $(66.6 \pm 4.5 \mathrm{yr})$ and the young participants $(\mathrm{n}=46)$ were ages $18-30 \mathrm{yr}(23.4 \pm 3.8 \mathrm{yr})$. Both age groups had low levels of depressed mood (CESD levels [25] of $8.6 \pm 1.0$ and $6.9 \pm 1.0$, respectively, for the young and older participants). No significant mood difference between age groups was found in laboratory levels of CES-D.

A significant mean post-treatment reduction in STAI was found $[\mathrm{F}(1,78)=17.92, \mathrm{p}<0.001]$. The corresponding effect size was 0.36 (95\% CI: 0.19-0.53). The reduction in STAI was significantly greater for participants with clinical levels of STAI (reduction of $12.37 \pm 6.880$ compared with those with lower STAI levels $(1.8 \pm 0.40)[\mathrm{t}(1,770=4.76$, $\mathrm{p}<\mathrm{v} 0.001]$. The anxiolytic effect was also observed separately for each of the three DAYS ( $\mathrm{p}=0.04,0.02,<0.001$, respectively). The corresponding effect sizes (and 95\% CI) were $0.23(0.01-0.45), 0.20(0.02-0.38)$, and $0.38(0.22-$ $0.54)$, respectively.

There was no significant age or sex effect. STAI data, collapsed across sex and age group are displayed in Figure 1.

\section{Discussion}

The results indicate a significant anxiolytic effect of acute bright light exposure. Moreover, the effect was consistent for each of the three days. The anxiolytic effect did not vary significantly by age group or sex of the participants. The corresponding effect size is considered small [27], but

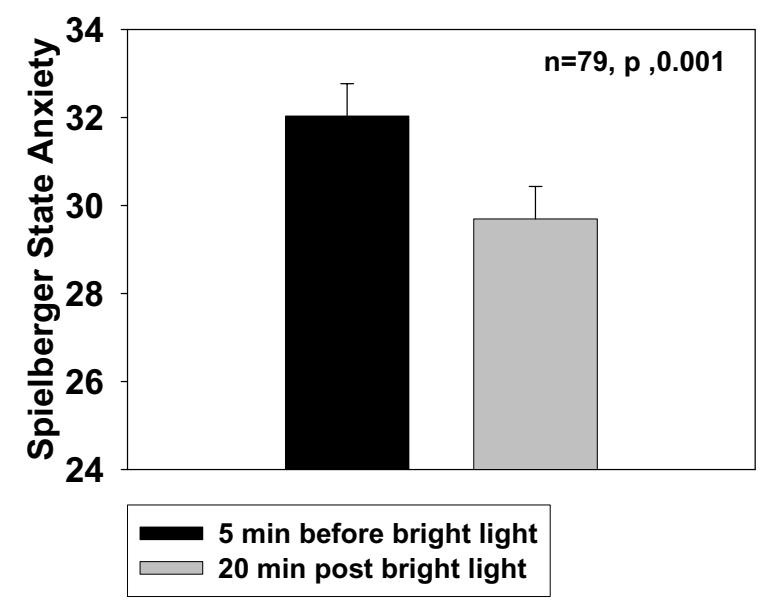

Figure I

Mean ( \pm SE) state anxiety (STAI-YI) assessed 5 min before and 20 min after bright light treatment ( $3 \mathrm{hr}$ at 3,000 lux) averaged over three consecutive days. Data are combined across age and sex.

comparable to other anxiolytic stimuli in low anxious adults $[28,29]$.

The data are consistent with other research suggesting a reduction of anxiety symptoms following bright light treatment of winter depression [20]. A unique aspect of the present study is that the anxiolytic effects were observed after acute exposure to bright light, whereas others reports had considered effects after 2 or more weeks of light treatment.

The results are not consistent with a recent study by Goel and Etwaroo [30], who found no significant effect of acute bright light treatment on the POMS-tension subscale, but a significant antidepressant effect was observed. The discrepancy in findings might be attributed to methodological differences. Whereas the present study involved $3 \mathrm{hr}$ of exposure to 3,000 lux light and assessment of anxiety change at $20 \mathrm{~min}$ post-exposure, the Goel and Etwaroo study examined anxiety changes during exposure to 30 min of 10,000 lux light [30].

There were several noteworthy limitations of the present study. First, an obvious methodological limitation was the lack of a control group, which was not possible in this ancillary open trial. Nonetheless, we do not think that the observed changes in STAI can be readily attributed to behavioral artifacts, such as demand characteristics or expectancy effects. Anecdotally, it seemed that the subjects viewed completion of the STAI as a small part of a complex study. As much focus was placed on following the 
ultra-short sleep-wake schedule, collection of urine samples, etc., it seemed that subjects were unaware of the research hypothesis of this ancillary study. Were demand or expectancy effects operating, greater anxiolytic effects might be expected. The STAI changes are likely also not attributable to experimentally imposed "time-out" from daily stressors, as the bright light treatment was not administered until the participants were in the laboratory for $=30 \mathrm{hr}$. Nonetheless, controlled, randomized studies are needed to verify these findings.

A second limitation was the low baseline levels of anxiety of the participants. As expected, the few high-anxious participants had far greater anxiolytic responses than the other participants. Despite receiving recommended standardized instructions for completing the STAI, a remarkably high number of participants were excluded from the analyses due to low baseline STAI levels $(<25)$. The decision to exclude these individuals was made a priori. The reasons for the low baseline STAI levels are unclear. The participants might have made efforts to complete the STAI questionnaires in a socially desirable manner. However, similarly abnormally low values were not observed for the CES-D data. The lack of inclusion of individuals ages 31$58 \mathrm{yrs}$, who tend to have high rates of anxiety, might have contributed to low baseline levels.

A third limitation was that the high levels of health and fitness of the participants was not representative of the population, particularly not that of older individuals. However, this limitation might have led to an underestimation of the efficacy of bright light, which have been more clearly demonstrated in less healthy individuals. Some correlates of health and fitness, such as better psychological health and greater habitual exposure to bright outdoor light, might have attenuated the anxiolytic effects of bright light.

A fourth limitation might have been the unique laboratory environment for exploring psychological benefits of bright light, involving prolonged maintenance of the ultra-short sleep-wake cycle, a moderate degree of sleep deprivation, low levels of light exposure, etc. However, despite the rigors of the experiment, it did not appear to be "stressful". For example there were no changes in pretreatment STAI levels between DAYs 1-3. Indeed, many subjects reported that the experience was calming. As addressed above, by separating the subjects from their usual daily stressors, the environment might have resulted in an underestimation of the anxiolytic effects of bright light treatment.

That participants had generally had low levels of depression suggests that the anxiolytic effect of light exposure was independent of significant antidepressant effects.
Moreover, in post-hoc analysis, we found no significant correlation of anxiolytic effect with baseline level of depression $(\mathrm{r}=0.043)$.

\section{Conclusion}

Notwithstanding its limitations, the present study provides provocative data addressing an important, but neglected area of research. Although anxiety and depression are the most common mental disorders, far more research focus has been placed on depression. This limitation is certainly true for bright light treatment. Whereas the antidepressant effects of bright light have been examined in dozens of studies, the studies did not focus on potential anxiolytic effects of bright light. That significant anxiolytic effects were observed following acute exposure in low-anxious subjects gives rise to the exciting possibility that far greater effects of bright light might be observed following chronic treatment in high-anxious individuals. Further randomized, controlled experiments are needed.

\section{Competing interests}

The author(s) declare that they have no competing interests.

\section{Authors' contributions}

SDY conceived the idea, directed collection of the data, and drafted the manuscript. DFK helped interpret the results and write the manuscript. All authors read and approved the manuscript.

\section{Acknowledgements}

The study was supported by grants AGI5763, HL6I280, and VA (VISN-7) Career Devlopment Award. KMR, ACC, PMO'B, JdR, and AMG assisted with this study.

\section{References}

I. Kessler RC, McGonagle KA, Zhao S, Nelson CB, Hughes M, Eshleman $S$, Wittchen $\mathrm{HO}$, Kendler KS: Lifetime and I 2-month prevalence of DSM-III-R psychiatric disorders in the United States: results from the National Comorbidity Survey. Arch Gen Psychiat 1994, 5 I:8-19.

2. Regier DA, Narrow WE, Rae DS, Manderscheid RW, Locke BZ, Goodwin FK: The de facto US mental and addictive disorders service system : epidemiologic catchment area prospective I-year prevalence rates of disorders and services. Arch Gen Psychiat 1993, 50:85-94.

3. Balon R: Mood, anxiety, and physical illness: body and mind, or mind and body? Depression Anxiety 2006, 23:377-87.

4. Muller JE, Koen L, Stein DJ: Anxiety and medical disorders. Current Psychiat Rep 2005, 7:245-5I.

5. Scott KM, Bruffaerts R, Tsang A, Ormel J, Alonso J, Angermeyer MC, Benjet C, Bromet E, de Girolamo G, de Graaf R, Gasquet I, Gureye O, Haro JM, He Y, Kessler RC, Levinson D, Mneimnah ZN, Oakley Browne MA, Posada-Villa JA, Stein DJ, Takeshima T, Van Korff M: Depression-anxiety relationships with chronic physical conditions: Results from the World Mental Health surveys. J Affect Disord 2007, 103(I-3): I I3-I 20.

6. Hoffman DL, Dukes EM, Wittchen HU: Human and economic burden of generalized anxiety disorder. Depress Anxiety 2006 in press. [Epub ahead of print]

7. Sanderson K, Tilse E, Nicholson J, Oldenburg B, Graves N: Which presenteeism measures are more sensitive to depression and anxiety? J Affect Disord 2007, I 01 :65-74. 
8. Buffett-Jerrott SE, Stewart SH: Cognitive and sedative effects of benzodiazepine use. Curr Pharm Des 2002, 8:45-58.

9. Beracochea $D$ : Anterograde and retrograde effects of benzodiazepines on memory. ScientificWorldJournal 2006, 6: | 460-| 465.

10. Struzik L, Vermani M, Coonerty-Femiano A, Katzman MA: Treatments for generalized anxiety disorder. Expert Rev Neurotherapeut 2004, 4:285-94.

II. Segraves RT: Sexual side-effects of psychiatric drugs. Intl J Psychiatry Med 1988, I 8(3):243-252

12. Rosenthal NE, Sack DA, Carpenter C), Parry B, Mendelson WB, Wehr TA: Antidepressant effects of light in seasonal affective disorder. Am J Psychiat 1985, 142:163-70.

13. Lewy AJ, Leflur BJ, Emens JS, Bauer VK: The circadian basis of winter depression. Proc Natl Acad Sci 2006, 103:74I 4-9.

14. Parry BL, Mahan AM, Mostofi N, Klauber MR, Lew GS, Gillin JC: Light therapy of late luteal phase dysphoric disorder : an extended study. Am J Psychiat 1993, 1 50:| 417-385.

15. Terman M, Terman JS: Light therapy for seasonal and nonseasonal depression: efficacy, protocol, safety, and side effects. CNS Spectrums 2005, 10:647-63.

16. Tuunainen A, Kripke DF, Endo T: Light therapy for non-seasonal depression. Cochrane Database Syst Rev 2004, 2:CD004050.

17. Epperson CN, Terman M, Terman JS, Hanusa BH, Oren DA, Peind KS, Wisner KL: Randomized clinical trial of bright light therapy for antepartum depression: preliminary findings. J Clin Psychiatry 2004, 65(3):42I-425.

18. Pollack MH: Comorbid anxiety and depression. J Clin Psychiatry 2005, 66(Suppl 8):22-29.

19. Sheehan DV, Eaddy MT, Shah MB, Mauch RP: Differences in total medical costs across the SSRIs for the treatment of depression and anxiety. Am J Manag Care 2005, I I (I 2 Suppl):S354-S36 I.

20. Terman M: On the specific action and clinical domain of light treatment. In Seasonal Affective Disorder and Beyond: Light Treatment for SAD and Non-SAD Conditions Edited by: Lam RW. Washington, DC: American Psychiatric Press, Inc; 1998:91-116.

21. Partonen T, LÖnnqvist J: Bright light improves vitality and alleviates distress in healthy people. J Affect Disord 2000, 57:55-6I.

22. Tam EM, Lam RW, Yatham LN, Zis AP: Psychobiological effects of light therapy in seasonal affective disorder. In Seasonal Affective Disorder and Beyond: Light Treatment for SAD and Non-SAD Conditions Edited by: Lam RW. Washington, DC: American Psychiatric Press, Inc; 1998: 1 17-42.

23. ACSM's Guidelines for Exercise Testing and Prescription Edited by: Franklin BA. Philadelphia: Lippicott Williams \& Wilkins; 2006.

24. Lavie P, Scherson A: Ultrashort sleep-waking schedule. I. Evidence of ultradian rhythmicity in 'sleepability'. Electroencephalogr Clin Neurophysiol 1981, 52:163-74.

25. Radloff LS: The CES-D scale : A self-report depression scale for research in the general population. Appl Psychol Measure | 977, I:385-40|.

26. Spielberger CD, Gorsuch RL, Lushene R, Vagg PR, Jacobs GA: Manual for the State-Trait Anxiety Inventory. Palo Alto : Consulting Psychologists Press; 1983.

27. Cohen J: Statistical Power Analysis for the Behavioral Sciences 2nd edition. Hillsdale, NJ: Lawrence Erlbaum

28. Petruzzello SJ, Landers DM, Hatfield BD, Kubitz KA Salazar W: A meta-analysis on the anxiety-reducing effects of acute and chronic exercise. Outcomes and mechanisms. Sports Med I99|, I I(3): |42-182.

29. Lehrer PM, Carr R, Sargunaraj D, Woolfolk RL: Stress management techniques: are they all equivalent, or do they have specific effects? Biofeedback Self Regul 1994, 19(4):353-40I.

30. Goel N, Etwaroo GR: Bright light, negative air ions and auditory stimuli produce rapid mood changes in a student population: a placebo-controlled study. Psychol Med 2006, 36:1253-63.

\section{Pre-publication history}

The pre-publication history for this paper can be accessed here:

http://www.biomedcentral.com/1471-244X/7/62/pre pub
Publish with Bio Med Central and every scientist can read your work free of charge

"BioMed Central will be the most significant development for disseminating the results of biomedical research in our lifetime. "

Sir Paul Nurse, Cancer Research UK

Your research papers will be:

- available free of charge to the entire biomedical community

- peer reviewed and published immediately upon acceptance

- cited in PubMed and archived on PubMed Central

- yours - you keep the copyright
BioMedcentral 